\title{
Visualizing non-classical formation pathways of alloyed nanocrystals with liquid phase transmission electron microscopy
}

\author{
Mei Wang ${ }^{1}$, Asher Leff ${ }^{2},{\text { Yue } \mathrm{Li}^{1} \text { and Taylor Woehl }}^{1}$ \\ ${ }^{1}$ University of Maryland, United States, ${ }^{2}$ Army Research Laboratory, United States
}

Liquid phase transmission electron microscopy (LP-TEM) has enabled unprecedented insights into the crystallization mechanisms of solution phase inorganic and organic nanoparticles [1-3]. Most LP-TEM studies of nanoparticle formation utilize the electron beam as a stimulus for nanoparticle formation. Generally, a solution phase inorganic or organic metal precursor is reduced by radicals created by electron beam radiolysis of the solvent $[3,4]$. While LP-TEM has yielded many high impact studies on nanocrystal formation, the question remains whether radiation chemistry drives reaction pathways similar to flask synthesis using chemical reducing agents. This is especially critical when using complex precursor ions, such as single source bimetallic precursors, whose molecular structure impacts the final morphology and atomic structure of the nanocrystals. It is known that damaging radical species are formed by the electron beam, such as hydroxyl radicals, which oxidize metal atoms and polymers. The question of electron beam damage and reaction chemistry during electron beam synthesis must be addressed if LP-TEM experiments are expected to produce useful insights that will guide synthesis of nanocrystals.

Alloying gold and copper together in nanoparticles is notoriously difficult because gold chloride precursor reduces more rapidly than copper ions. Recent work has explored the use of bimetallic precursor-ligand complexes for promoting alloying and have shown these complexes enhance alloying of gold and copper [5]; however, the alloying mechanism remains unclear. In this study we explore non-classical formation mechanisms of alloyed $\mathrm{AuCu}$ nanocrystals using LP-TEM. Prior to exploring nanocrystal formation mechanisms, we established ranges of LP-TEM electron beam synthesis (e.g. precursor concentration, magnification, beam current, dose rates) where the final nanocrystals had similar characteristics to flask synthesized nanocrystals. We performed high resolution TEM (HRTEM) and energy dispersive x-ray spectroscopy (EDS) composition measurements of the LP-TEM synthesized nanocrystals after they were removed from the liquid cell and dried and compared them to nanocrystals formed by aqueous sodium borohydride reduction in a flask (Figure 1). These experiments showed that nanocrystals synthesized by LP-TEM at a dose rate $<20 \mathrm{MGy} / \mathrm{s}$ had statistically indistinguishable alloy composition ( $\sim 55$ atomic\% $\mathrm{Au})$ and had similar particle size distribution and aggregation state. Nanocrystals formed at larger dose rates were significantly aggregated and were gold rich with little copper integration ( $<5$ atomic\%). Reaction kinetic simulations showed that dose rates $>20 \mathrm{MGy}$ significantly degraded the thiol capping ligand. These results indicate that the capping ligand is critical to form unaggregated nanocrystals as well as alloys containing significant amounts of copper [6]. 

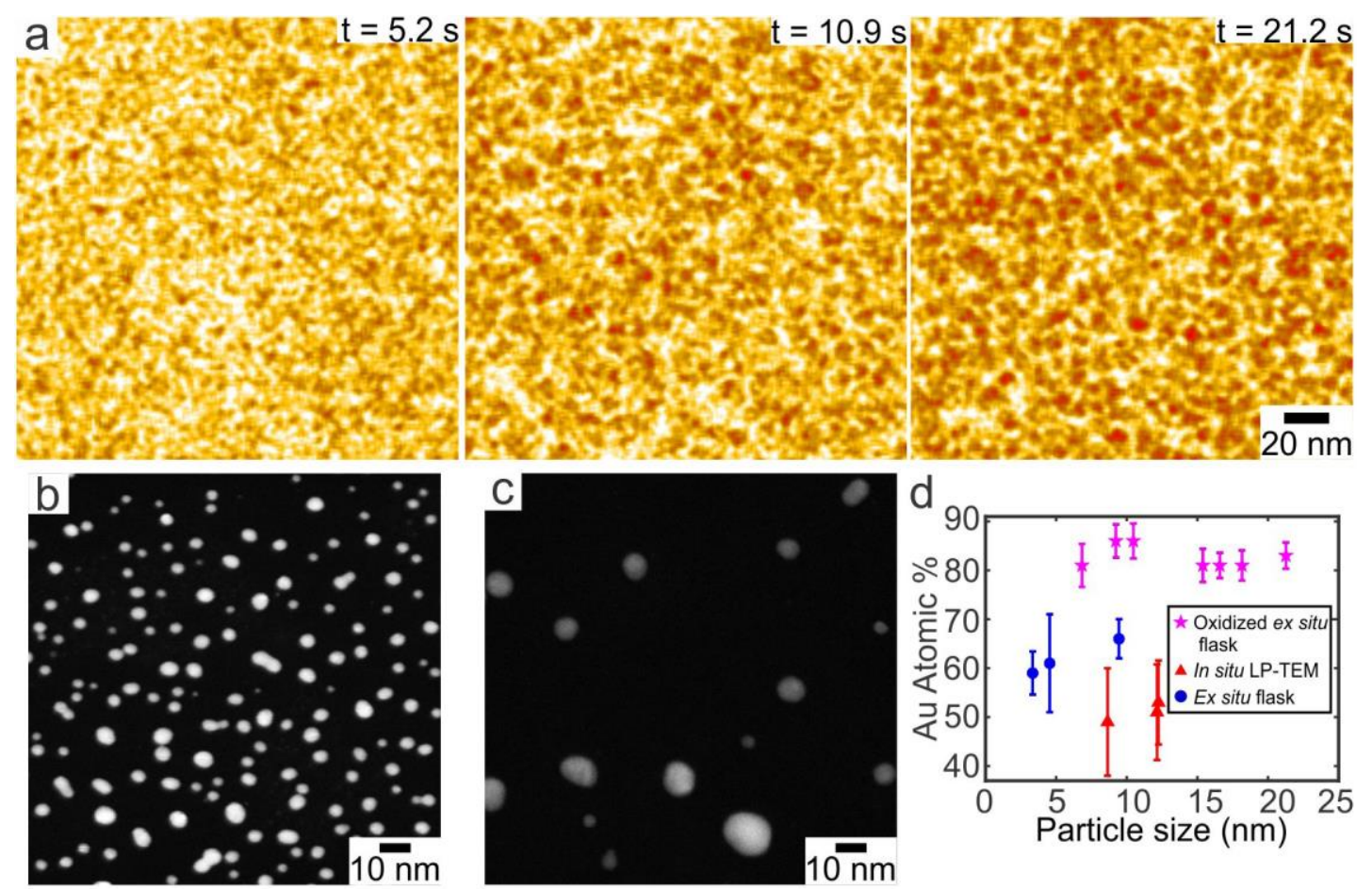

Figure 1. Figure 1. (a) False colored LP-TEM time lapsed series of images of AuCu nanocrystal formation taken at $\mathrm{M}=500,000$ and beam current of $16 \mathrm{pA}$ (dose rate =16.7 MGy/s). (b) HAADF-STEM image of $\mathrm{AuCu}$ nanocrystals formed by flask synthesis (b) and LP-TEM (c). (d) STEM-EDS measurements of $\mathrm{AuCu}$ nanocrystals formed by flask synthesis, LP-TEM synthesis, and flask synthesis from oxidized precursor.

\section{References}

[1] Zheng, H. M., Smith, R. K., Jun, Y. W., et al., Science, 324 (2009), p. 1309-1312.

[2] Patterson, J. P., Abellan, P., Denny, M. S., et al., J. Am. Chem. Soc., 137 (2015), p. 7322-7328.

[3] Woehl, T. J., Evans, J. E., Arslan, L., et al., Acs Nano, 6 (2012), p. 8599-8610.

[4] Woehl, T. J., Abellan, P., J. Microsc., 265 (2017), p. 135-147.

[5] Marbella, L. E., Chevrier, D. M., Tancini, P. D., J. Am. Chem. Soc., 137 (2015), p. 15852-15858.

[6] We acknowledge funding support from ACS PRF (\#61111-DNI10). 